\title{
Angiosarcoma Presenting with Minor Erythema and Swelling
}

\author{
Catherine A. Cox ${ }^{a}$ Richard O. Wein ${ }^{b}$ Roya Ghafouri ${ }^{d} \quad$ Nora M. Laver \\ Katrinka L. Heher ${ }^{a}$ Mitesh K. Kapadia ${ }^{a}$ \\ Departments of aphthalmology, ${ }^{\mathrm{b}}$ Otolaryngology, and ${ }^{\mathrm{c}}$ Pathology, Tufts Medical Center, \\ and ${ }^{\mathrm{d} D e p a r t m e n t}$ of Ophthalmology, Boston Medical Center, Boston, Mass., USA
}

\section{Key Words}

Tumor · Eyelid edema · Angiosarcoma · Eyelid cancer

\begin{abstract}
A 76-year-old man presented with slowly progressive swelling in his forehead and left upper eyelid over the course of three months. CT scanning showed non-specific enhancement of subcutaneous tissues, suggesting a low-grade cellulitis. Poor response to treatment prompted an MRI, which revealed the presence of a soft tissue lesion. Biopsy of this lesion was positive for angiosarcoma. The patient underwent chemotherapy and radiation, but unfortunately succumbed to his malignancy eight months later. This case illustrates a rare example of facial/periorbital angiosarcoma, a benign-appearing but aggressive tumor associated with a high incidence of mortality. A review of the literature and current treatment options are discussed.
\end{abstract}

\section{Introduction}

Angiosarcoma is a rare malignant neoplasm comprising $1 \%$ of all soft tissue sarcomas with a predilection for the skin and superficial soft tissues of the face and scalp [1]. Most lesions are diagnosed once the presence of an advanced ulcerated plaque or nodule has been identified. Early lesions, however, may mimic an infectious or inflammatory process, leading to a potential delay in diagnosis and treatment. We describe an unusual case of angiosarcoma presenting as isolated eyelid swelling, without a plaque or nodule, illustrating the sometimes benign clinical appearance of this very aggressive and deadly tumor. 
Cox et al.: Angiosarcoma Presenting with Minor Erythema and Swelling

\section{Case Description}

A 76-year-old man presented with slowly progressive swelling in his left upper eyelid for three months. He denied any pain, trauma, fever, eyelid lesions, or sinus disease. His past medical history was non-contributory. He had quit smoking fifty years prior.

On exam, best corrected visual acuity was 20/40 OU. No afferent pupillary defect, diplopia, or proptosis was present. External examination revealed an area of red, indurated skin near the glabella with soft tissue edema extending into the left upper lid (fig. 1). Slit lamp examination was unremarkable.

Computed tomography (CT) of the orbits demonstrated non-specific soft tissue changes corresponding to the areas of edema found on clinical examination (fig. 2). Cellulitis was suspected and oral cephalexin was started. The patient presented one week later with worsening forehead and upper eyelid swelling (fig. 1). Magnetic resonance imaging (MRI) revealed a soft tissue lesion lateral to the left orbit that extended medially into the midforehead (fig. 2). A diagnostic biopsy was performed, revealing poorly differentiated pleomorphic tumor cells with mitotic activity and focal slit-like vascular formation infiltrating the deep dermis. The tumor cells were immunoreactive to CD31 and factor 8 immunohistochemical staining, which was consistent with a diagnosis of angiosarcoma (fig. 3). Positron emission tomography (PET) scanning was negative for evidence of metastases.

Surgical resection of the tumor was discussed. The patient declined surgery due to the likelihood of significant morbidity from extensive tissue removal needed to clear the tumor margins. The patient underwent chemotherapy with docetaxel and bevacizumab, as well as radiation therapy to the affected region. Due to development of a grade IV skin reaction, the patient could only tolerate 50 Gy of a planned 60-Gy treatment. Additionally, bevacizumab was temporarily withheld due to proteinuria and then was later resumed at a reduced dose. Biopsies of the treatment field showed a partial response, but tumor metastasis was eventually found in the parotid gland and lungs. Despite treatment, the patient passed away eight months following initial diagnosis.

\section{Comment}

Our patient presented with deceptively benign findings given the aggressive nature of his tumor. An ecchymotic appearance suggested a vascular lesion, but lack of a mass on CT imaging made his disease process appear non-neoplastic.

Clinically, early angiosarcoma can present with mild erythema, facial edema and a bruised-like appearance. Longstanding lesions may show an overlying indurated plaque or nodular neoplasm associated with hemorrhage and ulceration [2]. Histologically, a welldifferentiated angiosarcoma is composed of dilated, anastomosing vascular channels lined by enlarged endothelial cells. Poorly differentiated lesions contain solid or sheath-like proliferations of polygonal or spindle-shaped endothelial cells [2]. A lymphocytic infiltrate may also be present, making it difficult to distinguish between angiosarcoma and other immune or inflammatory disorders [3].

Angiosarcoma has the potential to arise in any soft tissue of the body, however, cutaneous angiosarcoma is the most common presentation of which head and neck tumors account for up to $62 \%$ of cases [4]. Eyelid or periorbital lesions are reported infrequently [5-9]. Primary orbital lesions, as well as metastasis to the orbit, have been described in only a handful of cases [1]. 
Cox et al.: Angiosarcoma Presenting with Minor Erythema and Swelling

Age less than fifty, localization to the trunk or face, and size less than $5 \mathrm{~cm}$ are all good prognostic indicators $[4,10]$. Ultraviolet exposure is a presumed risk factor, although a direct correlation has not been proven [4]. Cutaneous angiosarcoma can be a rare complication of radiotherapy, and visceral organ tumors have been linked to environmental toxin exposure [1, 4]. Survival rates for head and neck angiosarcomas range from 10 to $51 \%$ at 10 years [4].

Ideally, smaller lesions are treated with complete wide local excision followed by aggressive chemoradiation [10]. In advanced cases with periorbital involvement, exenteration may be required $[3,10]$. Monotherapy with paclitaxel has been shown to be uniquely effective in reducing disease extent and prolonging disease-free intervals. Anti-VEGF agents have also been shown to be effective [11].

Given the size and ill-defined borders of his lesion, surgical resection was not performed in our patient's case. Attempts to control the tumor with chemotherapy and radiation were unfortunately unsuccessful. Angiosarcoma is an extremely rare tumor that is not often encountered by either ophthalmologists or oculoplastic surgeons. This tumor should, however, be considered in the differential diagnosis for any patient presenting with isolated eyelid swelling, particularly in the absence of infectious or inflammatory signs. Early diagnosis may lead to increased treatment options and a greater chance of survival.

\section{Acknowledgements}

This study was supported by a grant from the Research to Prevent Blindness to New England Eye Center.

\section{References}

-1 Penel N, Marréaud S, Robin YM, Hohenberger P: Angiosarcoma: state of the art perspectives. Crit Rev Oncol Hematol 2010; doi: 10.1016/j.critrevonc.2010.10.007.

2 Mentzel T: Sarcomas of the skin in the elderly. Clin Dermatol 2011;29:80-90.

3 Requena L, Santonja C, Stutz N, Kaddu S, Weenig RH, Kutzner H, Menzel T, Cerroni L: Pseudolymphomatous cutaneous angiosarcoma: a rare variant of cutaneous angiosarcoma readily mistaken for cutaneous lymphoma. Am J Dermatopathol 2007;29:342-350.

-4 Albores-Saavedra J, Schwartz AM, Henson DE, Kostun L, Hart A, Angeles-Albores D, Chablé-Montero F: Cutaneous angiosarcoma. Analysis of 434 cases from the Surveillance, Epidemiology, and End Results Program, 1973-2007. Ann Diagn Pathol 2010; doi: 10.1016/j.anndiagpath.2010.07.012.

5 de Keizer RJ, de Wolff-Rouendaal D, Nooy MA: Angiosarcoma of the eyelid and periororbital region. Experience in Leiden with iridium 192 brachytherapy and low-dose doxorubicin chemotherapy. Orbit 2008;27:5-12.

6 DeMartelaere SL, Roberts D, Burgess MA, Morrison WH, Pisters PW, Sturgis EM, Ho V, Esmaeli B: Neoadjuvant chemotherapy-specific and overall treatment outcomes in patients with cutaneous angiosarcoma of the face with periorbital involvement. Head Neck 2008;30:639-646.

7 Gündüz K, Shields JA, Shields CL, Eagle RC Jr, Nathan F: Cutaneous angiosarcoma with eyelid involvement. Am J Ophthalmol 1998;125:870-871.

$\checkmark 8$ Papalas JA, Manavi CK, Woodward JA, Sangueza OP, Cummings TJ: Angiosarcoma of the eyelid: a clinicopathologic comparison between isolated unilateral tumors and tumors demonstrating extrapalpebral involvement. Am J Dermatopathol 2010;32:694-699.

-9 Wiwatwongwana D, White VA, Dolman PJ: Two cases of periocular cutaneous angiosarcoma. Ophthal Plast Reconstr Surg 2010;26:365-366.

10 Guadagnolo BA, Zagars GK, Araujo D, Ravi V, Shellenberger TD, Sturgis EM: Outcomes after definitive treatment for cutaneous angiosarcoma of the face and scalp. Head Neck 2010; doi: 10.1002/hed.21513.

-11 Fuller CK, Charlson JA, Dankle SK, Russell TJ: Dramatic improvement of inoperable angiosarcoma with combination paclitaxel and bevacizumab chemotherapy. J Am Acad Dermatol 2010;63:e83-e84. 
Case Reports in

Ophthalmology
Case Rep Ophthalmol 2013;4:59-63

DOI: $10.1159 / 000346952$

Cox et al: Angiosarcoma Presenting with Minor Erythema and Swelling
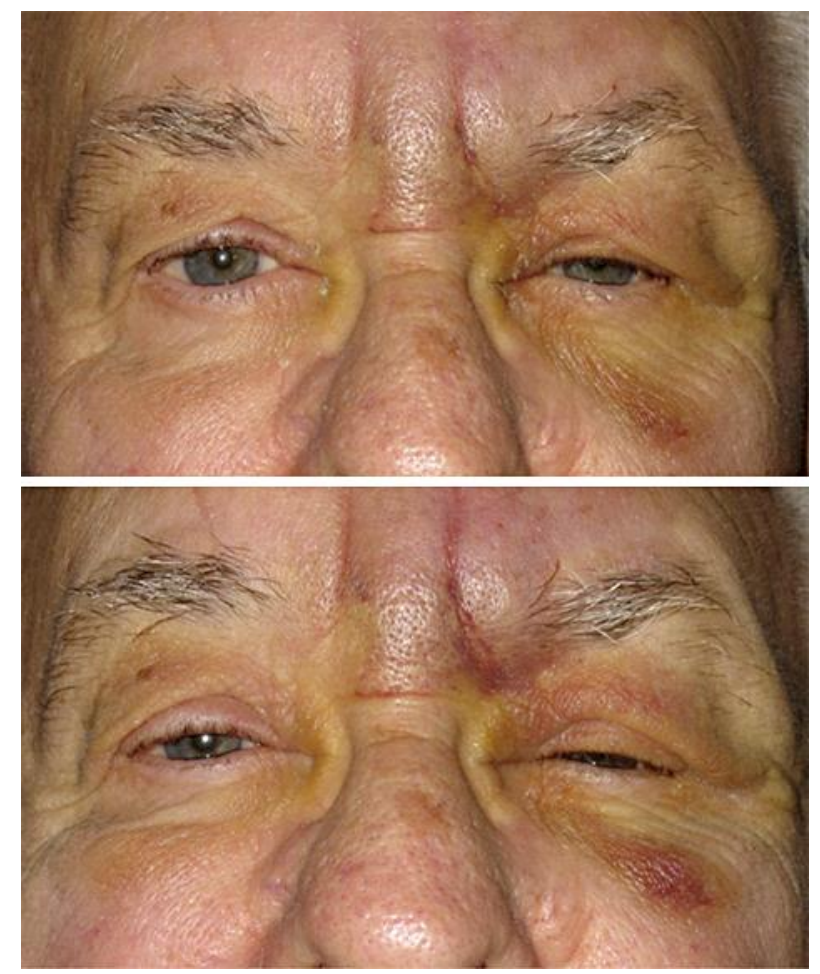

Fig. 1. Top: Mild periorbital erythema and left upper lid swelling were present on initial presentation Bottom: One week later, periorbital erythema and left upper lid swelling have become more pronounced. 

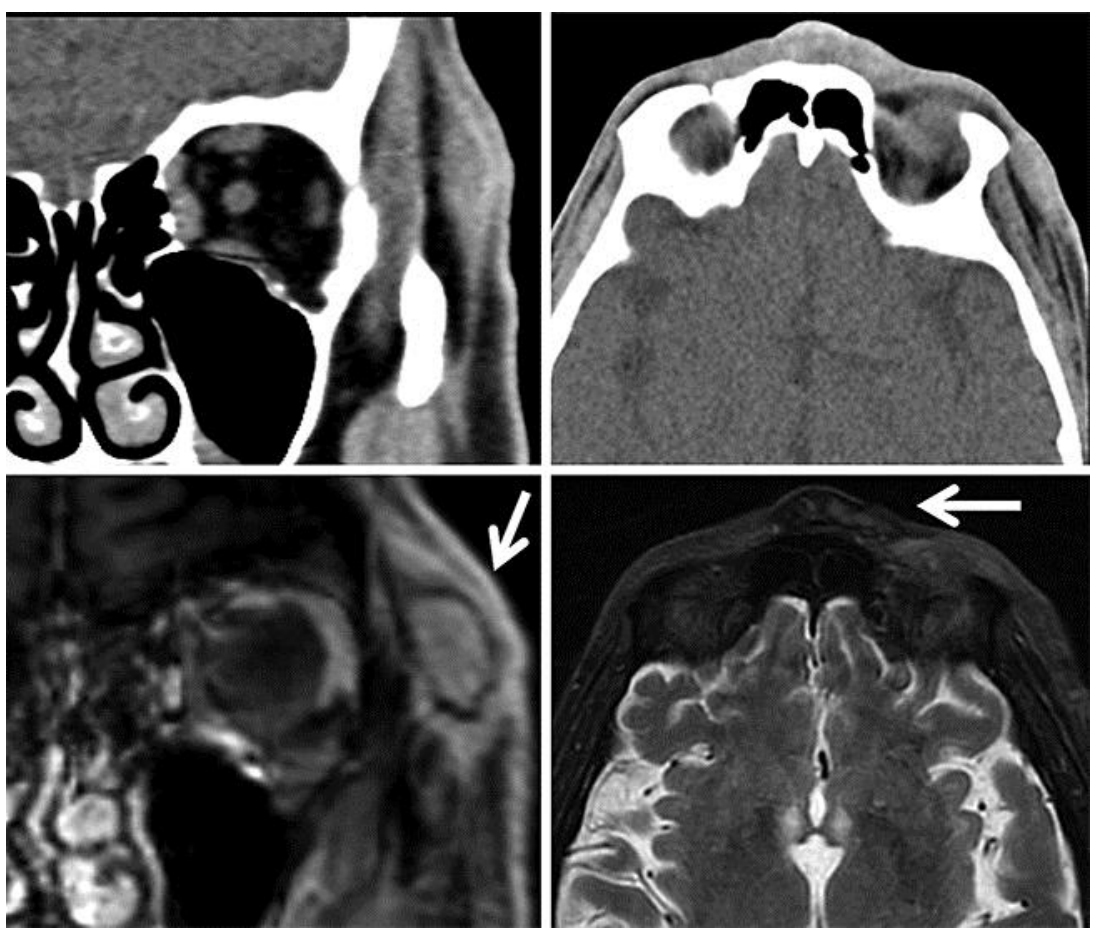

Fig. 2. Top: CT scan. Non-specific soft tissue swelling temporal to the left orbit (left) and in the glabellar region (right). Bottom: T1-weighted MRI, post-gadolinium. A distinct soft tissue lesion is visible superficial to the left temporalis muscle and orbit (left, arrow), as well as in the glabellar region (right, arrow). Enhancement of adjacent subcutaneous tissues and fascia is also present.
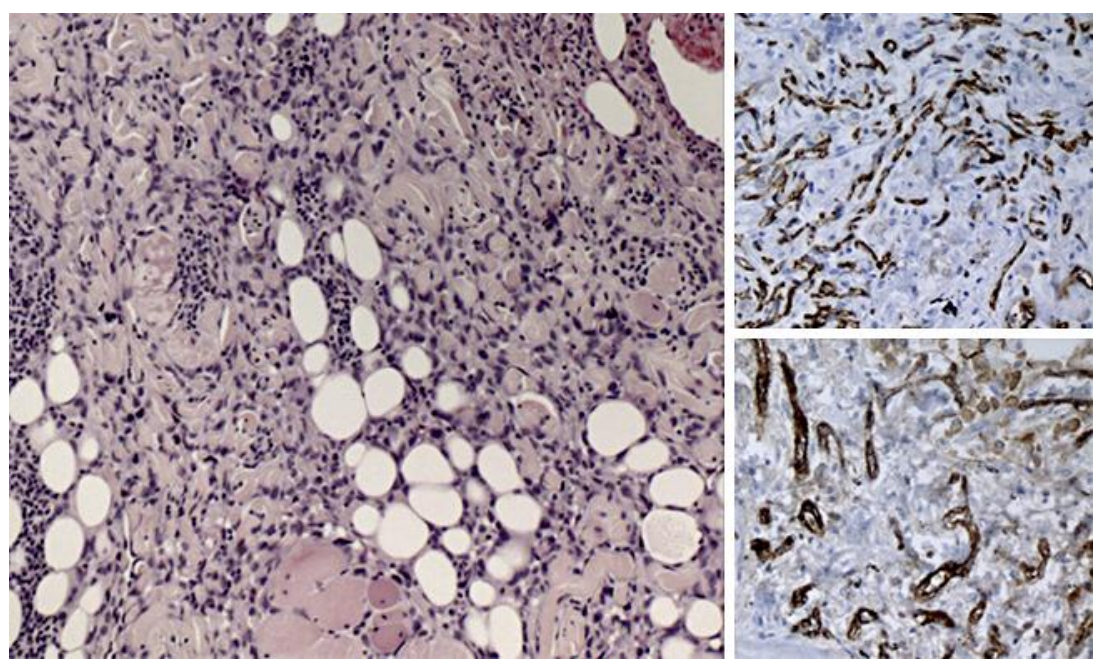

Fig. 3. Histopathological examination of tissue biopsy. Poorly differentiated tumor cells infiltrating the deep dermis with rudimentary vascular lumen formation (left). Antibody stains for CD31 (top right) and Factor 8 (bottom right) were both positive for vascular endothelial tissue, confirming the diagnosis of angiosarcoma. 